\title{
WEAK EXPECTATIONS AND INJECTIVITY IN OPERATOR ALGEBRAS
}

\author{
BRUCE E. BLACKADAR
}

\begin{abstract}
An example is given of a noninjective von Neumann algebra $\boldsymbol{M}$ on a separable Hilbert space $\mathcal{H}$ for which there exists a weak expectation of $\mathcal{L}(\mathcal{H})$ into $M$. Some positive results about weak expectations are also obtained.
\end{abstract}

1. Introduction. Much of the recent work in the structure theory of $C^{*}$-algebras and von Neumann algebras has been concerned with the closely related concepts of nuclearity and injectivity (see [3], [4]). It is becoming evident that the classes of nuclear $C^{*}$-algebras and injective von Neumann algebras have a simple enough structure to be tractable (at least in the separable case), while being broad enough to include most algebras of interest.

Lance [7] introduced the concept of a weak expectation, and showed its close connection with the problem of extending cross norms on tensor products of $C^{*}$-algebras. Subsequently, Choi and Effros [2] made a more detailed study of weak expectations and injectivity. Lance raised a number of important questions, one of which (slightly rephrased) is the following:

Question. Let $M$ be a von Neumann algebra on a Hilbert space $\mathcal{H}$. If there is a weak expectation for $M$, is $M$ necessarily injective?

Choi and Effros [2], using a result of Wassermann [11], gave a negative answer if $\mathcal{H}$ is not separable. But in view of results such as those of [4], [5], and $\$ 2$ of this paper, it is reasonable to expect the situation for a separable $\mathcal{H}$ to be nicer.

Nonetheless, the answer to Lance's question is negative, even if $\mathcal{H}$ is separable; a counterexample is given in $\$ 3 . \$ 4$ is a discussion of open questions and applications.

Most of the research for this paper was conducted at a Research Institute for Operator Theory at the University of New Hampshire, Summer 1976, funded by a grant from the National Science Foundation. I would also like to thank Edward Effros for valuable discussions on the subject of this paper.

2. Positive results. In this section, we describe some of the structure associated with weak expectations, particularly when the underlying Hilbert space is separable.

Received by the editors February 2, 1977 and, in revised form, May 19, 1977.

AMS (MOS) subject classifications (1970). Primary 46L10, 46L05.

Key words and phrases. $C^{*}$-algebra, von Neumann algebra, conditional expectation. 
Throughout this section, let $A$ be a $C^{*}$-algebra of operators on a Hilbert space $\mathcal{H}$, containing the identity, and let $M=A^{\prime \prime}$ be its weak closure. Recall that a weak expectation for $M$ with respect to $A$ is a completely positive map $P$ from $\mathcal{L}(\mathcal{F C})$ into $M$ such that $P(1)=1$ and $P(a x)=a P(x)$ and $P(x a)=$ $P(x) a$ for all $a \in A, x \in \mathcal{L}(\mathcal{H})$. If $A=M, P$ is called a conditional expectation, and $M$ is said to be injective if there is a conditional expectation of $\mathcal{L}(\mathcal{H})$ onto $M$. Details can be found in [2], [7], and [10].

Unlike conditional expectations, weak expectations need not be idempotent; however, the next theorem shows that idempotent weak expectations can always be found.

THEOREM 2.1. If there is a weak expectation for $M$ with respect to $A$, there is an idempotent weak expectation for $M$ with respect to $A$.

Proof. Let $\delta$ be the set of all weak expectations for $M$ with respect to $A$. $\mathcal{S}$ is a compact convex subset of $\mathscr{L}(\mathscr{L}(\mathcal{H})$ ) with the topology of pointwise $\sigma$-weak convergence. Let $P_{0} \in \mathcal{S}$; then the function $P \mapsto P \circ P_{0}$ is a continuous map of $\delta$ into $\delta$, and so has a fixed point $P_{1}$ by the Schauder fixed point theorem. Similarly, there is a $P_{2} \in \delta$ such that $P_{2} \circ P_{1}=P_{2}$. Then $P_{2} \circ P_{0}=\left(P_{2} \circ P_{1}\right) \circ P_{0}=P_{2} \circ\left(P_{1} \circ P_{0}\right)=P_{2} \circ P_{1}=P_{2}$. Continuing inductively, we construct a sequence $\left\{P_{n}\right\}$ such that $P_{n} \circ P_{m}=P_{n}$ if $n>m$. Let $P_{\omega}$ be any limit point of the sequence $\left\{P_{n}\right\}$; then $P_{\omega} \circ P_{n}=P_{\omega}$ for all $n$. Thus the construction can be continued transfinitely to give a $P_{\alpha} \in \mathcal{S}$ for each ordinal $\alpha$, such that $P_{\alpha} \circ P_{\beta}=P_{\alpha}$ if $\alpha>\beta$. Eventually, the transfinite sequence must repeat.

For the rest of the section, we will assume that $P$ is an idempotent weak expectation for $M$ with respect to $A$. We may assume that $A=\{a \in M$ : $P(a x)=a P(x), P(x a)=P(x) a \forall x \in \mathcal{L}(\mathcal{H})\}$. Let $R$ be the range of $P$. Then $A \subseteq R \subseteq M$, and by [1, Theorem 3.1], $A=\left\{a \in R: a^{*} a \in R, a a^{*} \in\right.$ $R$ \}. Thus, $A$ contains all projections of $R$, and if $R$ is a Jordan algebra, then $A=R$ (so $R$ is an algebra). By [2, Theorem 3.1] and [10, Theorem 5], $R$ becomes a conditionally complete injective $A W^{*}$-algebra under the multiplication $x \cdot y=P(x y)$.

The next proposition shows that if $\mathcal{H}$ is separable, then "most" spectral projections of selfadjoint elements of $A$ are again in $A$.

Proposition 2.2. Suppose $\mathcal{H}$ is separable. Let $x \in A, x=x^{*}$. Then $x$ is in the $C^{*}$-subalgebra of $A$ generated by the spectral projections of $x$ which are in A.

Proof. Let $\lambda, \mu \in \mathbf{R}, \lambda<\mu, \lambda$ and $\mu$ not in the point spectrum of $x$. Then $E_{[\lambda, \mu]}(x)=E_{(\lambda, \mu)}(x)$ is a projection of $\mathcal{L}(\mathcal{H})$ which is both an increasing and a decreasing limit of elements of $A^{+}$. Since $P$ is order-preserving, $E_{[\lambda, \mu]}(x) \in R$. But every projection in $R$ is in $A$. Since the point spectrum of $x$ is countable, its complement is dense in $\mathbf{R}$.

COROLlary 2.3. If $\mathcal{H}$ is separable, every maximal commutative subalgebra of 
$A$ is generated by projections, and the projections of $A$ are strongly dense in the projections of $\boldsymbol{M}$.

Proof. Let $e$ be a projection of $M$, and let $\left\{x_{n}\right\}$ be a sequence of selfadjoint elements of the unit ball of $A$ with $x_{n} \rightarrow e$ strongly (Kaplansky density.) Let $\lambda_{n} \in[1 / 3,2 / 3] \sim \sigma_{p}\left(x_{n}\right)$, and let $e_{n}=E_{\left[\lambda_{n}, 1\right]}\left(x_{n}\right)$. Then $e_{n}$ is a projection in $A$, and $e_{n} \rightarrow e$ strongly.

REMARK. A similar proof shows that if $A$ is a conditionally complete $C^{*}$-algebra which has a faithful representation $\pi$ on a separable Hilbert space, then every maximal commutative $C^{*}$-subalgebra of $\pi(A)$ is generated by projections, and therefore $A$ is an $A W^{*}$-algebra.

If $\mathcal{H}$ is separable and $R$ is an algebra, in many cases we can conclude from [5] that the identity representation of $R$ is normal, i.e. the supremum in $R$ of an increasing sequence of projections of $R$ is the same as the supremum in $\mathcal{L}(\mathcal{H})$. In particular, if $M$ is a factor, then $R$ is an $A W^{*}$-factor, so [5, Theorems 1 and 2] apply. The following proposition then shows that $R=M$.

Proposition 2.4. Let $B$ be an $A W^{*}$-algebra, and let $\pi$ be a faithful normal representation of $B$. Then $B$ is $a W^{*}$-algebra, and $\pi(B)$ is weakly closed.

Proof. $B$ is a $W^{*}$-algebra by [8, p. 174]. Let $C$ be a maximal commutative subalgebra of $B ; C$ is a commutative $W^{*}$-algebra, and $\pi(C)$ is weakly closed by [9, 1.13.2 and 1.16.2]. Thus $\pi(B)$ is weakly closed by [8, p. 174].

We summarize the above results in a theorem.

THEOREM 2.5. Let $M$ be a factor on a separable Hilbert space. If there is an indempotent weak expectation $P$ for $M$ (with respect to some weakly dense $C^{*}$-subalgebra) whose range is a Jordan algebra, then $P$ is a conditional expectation onto $M$, and so $M$ is injective.

The following argument, due to E. Effros, indicates that the hypothesis that $\mathcal{H}$ be separable is probably necessary. Let $A$ be a hyperfinite $\mathrm{II}_{1}$ factor; then $A^{* *}$ is not injective ([11, Corollary 1.9] and [3, Theorem 3]), so there ought to be a direct summand $M$ of $A^{* *}$ which is a noninjective factor (if $A$ were separable, this would follow from [4, 6.4(b) and 6.5]; the difficulty in the present case is the lack of a direct integral theory). If $M$ is such a factor, let $\pi$ be a representation of $A$ on a Hilbert space $\mathcal{H}$ with $\pi(A)^{\prime \prime}=M . \pi$ is faithful since $A$ is simple; since $A$ is injective, there is a conditional expectation of $\mathcal{L}(\mathcal{H})$ onto $\pi(A)$, which is a weak expectation for $M$.

The hypothesis that $M$ be a factor is also necessary: there exists a faithful representation $\pi$ of $l^{\infty}$ on a separable Hilbert space $\mathcal{H}$ such that $\pi\left(l^{\infty}\right)$ is not weakly closed (W. Bade, unpublished). Since $l^{\infty}$ is injective, there is a conditional expectation of $\mathcal{L}(\mathcal{H})$ onto $\pi\left(l^{\infty}\right)$ which is a weak expectation for $M=\pi\left(l^{\infty}\right)^{\prime \prime}$.

3. Negative results. In this section we give an example of a noninjective von Neumann algebra $M$ on a separable Hilbert space which has a weak expectation with respect to a weakly dense $C^{*}$-subalgebra. 
Let $A=B=C^{*}\left(\mathrm{~F}_{2}\right)$, the group $C^{*}$-algebra of the free group on two generators. Let $\rho_{1}, \rho_{2}, \ldots$ (respectively $\sigma_{1}, \sigma_{2}, \ldots$ ) be pairwise nonequivalent irreducible representations of $A$ (resp. $B$ ) such that $\boxplus \rho_{i}$ (resp. $\boxplus \sigma_{i}$ ) is faithful. Let $\pi_{i}=\rho_{i} \otimes \sigma_{i}$ be the corresponding irreducible representation of $A \otimes B$ on $\mathcal{F}_{i}$ (throughout this section, "tensor product" will always mena "minimal tensor product", using the least $C^{*}$-cross norm). Let $\pi_{0}$ be the representation of $A \otimes B$ generated by the left and right regular representations of $F_{2}$. (By [11, Proposition 2.7] the regular representation actually gives a representation of the minimal tensor product.) Let $\pi_{0}$ act on $\mathcal{H}_{0}$, and let $M_{i}=\pi_{i}(A \otimes 1)^{\prime \prime}$ for each $i \geqslant 0$. Let $\mathcal{H}=\bigoplus_{i=0}^{\infty} \mathcal{K}_{i}, M=\bigoplus_{i=0}^{\infty} M_{i}$, $\pi=\bigoplus_{i=0}^{\infty} \pi_{i}$. Then $\pi$ is a faithful representation of $A \otimes B$, and $\pi(A \otimes 1)^{\prime \prime}=$ $\pi(1 \otimes B)^{\prime}=M$ since all of the $\rho_{i}$ 's and $\sigma_{i}$ 's are nonequivalent. As in the proof of [7, Theorem 3.3], we may regard $A \otimes B$ as a subalgebra of $\mathcal{L}(\mathcal{H C}) \otimes B$, and so $\pi$ extends to a representation $\hat{\pi}$ of $\mathcal{L}(\mathcal{H}) \otimes B$ on a larger Hilbert space $\mathcal{K}$. For $x \in \mathcal{L}(\mathcal{H})$, let $P(x)$ be the compression of $\hat{\pi}(x \otimes 1)$ to $\mathcal{H}$; then $P$ is a weak expectation of $\mathcal{L}(\mathcal{H C})$ into $M$ with respect to $\pi(A \otimes 1)$. However, it is well known that $M_{0}$ is not injective, so $M$ is not injective.

4. Open questions. An important feature of the weak expectation constructed in $\$ 3$ is that the weak expectation algebra for $P$ does not contain the center of $M$. The fact that $\pi(A \otimes 1)$ is skewed with respect to the center of $M$ seems essential for the construction. In fact, Lance [7, Theorem 4.2] has shown that there is no weak expectation for $M_{0}$ with respect to $\pi_{0}(A \otimes 1)$.

It is natural to rephrase the original question in the following form:

Question. Let $M$ be a von Neumann algebra on a separable Hilbert space. If there is a weak expectation for $M$ with respect to a weakly dense subalgebra containing the center of $M$, is $M$ necessarily injective?

Using direct integral theory, it is enough to settle the question for $M$ a factor.

If the answer to this question is yes, then a modification of the techniques of [7] can be used to prove the following two consequences, providing a solution to two of the most important open questions concerning nuclear $C^{*}$-algebras:

(1) Every separable $C^{*}$-algebra can be embedded into a separable nuclear $C^{*}$-algebra.

(2) There exists a separable, simple, purely infinite, nuclear $C^{*}$-algebra with identity; such a $C^{*}$-algebra cannot be an inductive limit of type I $C^{*}$-algebras by [6].

ADDED IN Proof. The above question and consequence (1) have been settled negatively by the author [12], and consequence (2) settled affirmatively by J. Cuntz [13].

\section{REFERENCES}

1. M.-D. Choi, A Schwartz inequality for positive linear maps on $C^{*}$-algebras, Illinois J. Math. 18 (1974), 565-574. 
2. M.-D. Choi and E. Effros, Injectivity and operator spaces, J. Functional Analysis 24 (1977), 156-209.

3. (1977), 443-446.

4. A. Connes, Classification of injective factors, Ann. of Math. 104 (1976), 73-115.

5. J. Feldman and J. M. G. Fell, Separable representations of rings of operators, Ann. of Math. 65 (1957), 241-249.

6. B. Johnson, Cohomology in Banach algebras, Mem. Amer. Math. Soc. No. 127, 1972.

7. C. Lance, On nuclear $C^{*}$-algebras, J. Functional Analysis 12 (1973), 157-176.

8. G. Pedersen, Operator algebras with weakly closed abelian subalgebras, Bull. London Math. Soc. 4 (1972), 171-175.

9. S. Sakai, $C^{*}$-algebras and $W^{*}$-algebras, Springer-Verlag, Berlin and New York, 1971.

10. J. Tomiyama, On the projection of norm one in $W^{*}$-algebras, Proc. Japan Acad. 33 (1957), $608-612$.

11. S. Wassermann, On tensor products of certain group $C^{*}$-algebras, J. Functional Analysis 23 (1976), 239-254.

12. B. Blackadar, Weak expectations and nuclear $C^{*}$-algebras, (preprint).

13. J. Cuntz, Simple $C^{*}$-algebras generated by isometries, (preprint).

Department of Mathematics, University of Nevada, Reno, Nevada 89557 